\title{
Sistema de monitoreo para pacientes con tratamientos de tendinosis del tendón rotuliano utilizando Kinect
}

\author{
Monitoring system for patients with treatment of tendinosis \\ of patellar tendon using Kinect \\ Jonathan Córdova-Guarachi $^{1} \quad$ Diego Aracena-Pizarro ${ }^{1} \quad$ Jorge Corrales-Muñoz $^{2}$ \\ Recibido 7 de mayo de 2015, aceptado 11 de enero de 2016 \\ Received: May 7, 2015 Accepted: January 11, 2016
}

\begin{abstract}
RESUMEN
Este trabajo presenta el desarrollo y análisis de un sistema de monitoreo a distancia para pacientes con tratamientos kinesiológicos de tendinosis del tendón rotuliano, que captura los movimientos del paciente mediante un dispositivo de captura de profundidad (KINECT TM). Dicho sistema permite tanto al kinesiólogo como a su paciente trabajar en un tratamiento de recuperación de forma tal que el médico tratante, tras consulta diagnóstica previa, defina un protocolo de ejercicios que el paciente deberá realizar en su casa y asistido con el sistema desarrollado, el que entre sus funciones permite al paciente corregir sus movimientos y generar una estadística para así determinar el grado de avance del paciente en su tratamiento de recuperación. La estadística generada por el sistema queda a disposición del kinesiólogo dentro de un subsistema de administración para un uso futuro.

Con el sistema desarrollado se realizaron pruebas que determinaron la precisión del sistema con resultados aceptables. Para ello se contó con la colaboración de alumnos de kinesiología de la Universidad de Tarapacá, quienes aportaron voluntariamente en las pruebas realizadas con el sistema contrastado con una técnica tradicional, para medir el progreso de los pacientes en tratamientos kinesiológicos.
\end{abstract}

Palabras clave: Cámara de profundidad (KINECT), visión computacional, interacción, realidad aumentada, kinesiología.

\begin{abstract}
This work presents the development and analysis of remote monitoring system for patients with kinesiological treatment of patellar tendon tendinosis, which captures the patient's moves through a depth capture device (KINECT TM). This system allows both the treating kinesiologist and his patient, to work on a recovery treatment so that the attending kinesiologist, after previous diagnostic consultation, is able to define a protocol of exercises for the patient to be performed at home, assisted by the developed system, which functions allow the patients to correct their movements and generate statistics to determine the degree of progress in the treatment. The statistics generated by the system is available to the kinesiologist within a management. Tests were performed with the developed system to assess its accuracy with acceptable results. For this, kinesiology students of Universidad de Tarapacá participated voluntarily, contributing with tests that contrasted the system with a traditional technique, so as to measure the progress of patients in kinesiological treatment.
\end{abstract}

Keywords: Depth camera (KINECT), computer vision, interaction, augmented reality, kinesiology.

\footnotetext{
1 Escuela Universitaria de Ingeniería Industrial, Informática y Sistemas. Área de Ingeniería en Computación e Informática. Universidad de Tarapacá. Arica, Chile. E-mail: daracena@uta.cl; jonathan.cordova@alumnos.uta.cl

2 Facultad de Ciencias de la Salud. Departamento de Kinesiología y Nutrición. Universidad de Tarapacá. Arica, Chile. E-mail: jcorrale@uta.cl
} 


\section{INTRODUCCIÓN}

La labor principal de los profesionales de la kinesiología radica en disminuir la discapacidad de todo individuo que sufra alguna enfermedad o dolencia, entregando un soporte fundamental en la recuperación parcial o total de las funciones motrices perdidas [1]. Es por ello que el kinesiólogo, además de diseñar una rutina de ejercicios específica para cada paciente, debe velar para que este consiga los movimientos adecuados que aseguren una recuperación efectiva. Para ello el profesional maneja diferentes técnicas que permiten obtener información acerca del desarrollo de la terapia, desde la observación por repetición, control del tiempo, al uso de instrumentos que miden parámetros específicos, permitiendo al profesional de la salud tomar una decisión respecto del progreso de la terapia.

Sin embargo, existen diversos factores que pueden definir el éxito o fracaso del tratamiento, como la relación kinesiólogo-paciente, factores socioeconómicos o el entorno de desarrollo de la terapia [2].

Por lo demás, la tecnología de realidad aumentada y la evolución tecnológica han impulsado en estos últimos años el desarrollo de aplicaciones y dispositivos más sofisticados y de costos cada vez más bajos, que generan nuevas formas de interactuar. Tal es el caso de los controles con sensores de movimiento de Nintendo® para su consola Nintendo Wii ${ }^{\mathrm{TM}}$ [3], o el sensor de captura de profundidad KINECT ${ }^{\mathrm{TM}}$ que Microsoft ${ }^{\circledR}$ desarrolló para la consola de videojuegos Xbox $360^{\mathrm{TM}}$ [4], cuyas principales características pueden trascender del ámbito del entretenimiento, al ser aplicadas en el desarrollo de otras áreas.

En el presente trabajo se propone el diseño, desarrollo y análisis de un sistema de monitoreo de bajo costo, que permita analizar los movimientos de pacientes frente a un ejercicio kinesiológico definido utilizando una cámara de profundidad KINECT para Xbox 360. Además de poner a prueba la precisión del sistema a desarrollar, contrastando sus resultados con técnicas tradicionales de extracción de datos que los kinesiólogos en ejercicio realizan con sus pacientes, para controlar la evolución de sus tratamientos.
Existe diversa literatura que da cuenta del uso de la cámara de profundidad KINECT en áreas similares a la kinesiológica.

En Perandones $[2,5]$ se describe que el uso de software tipo videojuego en hospitales puede:

- Reducir el estrés físico del paciente.

- Reducir la tensión psicológica del paciente.

- Reducir la ira del paciente.

- Aumentar el vigor del paciente frente al tratamiento del mismo.

- Reducir la fatiga del paciente frente al tratamiento del mismo.

En Freitas, De Gama y otros [6] se propuso el diseño de un juego basado en el uso del sensor KINECT para la rehabilitación motora. En dicha aplicación tipo videojuego el paciente realiza diferentes posturas de acuerdo con las reglas impuestas por la aplicación lúdica. Dichas posturas son analizadas por la aplicación y sus resultados se entregan al paciente en forma de puntaje.

Por otra parte en Muñoz, Henao y López [7] se muestra la construcción de un sistema de rehabilitación física de pacientes que mezcla dinámicas tipo videojuego y el uso de un software específico para registro y análisis de los datos de captura de movimiento, datos extraídos haciendo uso de un sensor KINECT.

Centrándose solo en la parte técnica, en Osorio, Vázquez, Luna [8] se presenta un sistema basado en el uso del sensor KINECT para realizar el análisis de la marcha. En este sistema se emplea el sensor como herramienta para capturar los pasos del sujeto de prueba, los datos capturados son procesados por un software de apoyo con el que se pretende buscar anormalidades en la secuencia de pasos dados por el paciente.

En cuanto al uso de realidad aumentada, es interesante tomar en consideración el trabajo propuesto en Arango, Cárdenas y Peña Palacio [9], el que propone un sistema para rehabilitar pacientes que presentan el síndrome del miembro fantasma, una condición mental que hace que el cerebro genere sensación de dolor a un miembro del cuerpo que ya no existe, debido a la amputación del mismo. En este sistema se utiliza realidad aumentaba basada en marcas 
y un sensor de ondas cerebrales. Las marcas son interpretadas por el sistema de realidad aumentada con el fin de superponer, de forma virtual, un modelo 3D del miembro amputado sobre la marca. A su vez, este modelo reaccionará de acuerdo a cómo el sistema interprete las señales cerebrales.

Este trabajo se centra en la elaboración de un prototipo que utilice de forma innovadora la cámara de profundidad KINECT, como dispositivo de captura y mapeo de profundidad de bajo costo, en los tratamientos de pacientes afectados por tendinosis del tendón rotuliano, una de las dolencias más frecuentes en el mundo deportivo [10], que permita al paciente desarrollar ejercicios excéntricos $[10,11]$ en su hogar, monitoreados a distancia por el kinesiólogo tratante. Para ello aprovecha las experiencias descritas [7] en cuanto a la aplicación de ejercicios lúdicos y el trabajo desarrollado por Osorio, Vázquez, Luna [8] en cuanto a cómo KINECT se utiliza para extraer parámetros del cuerpo humano.

\section{ARQUITECTURA DEL SISTEMA}

Para el diseño de la arquitectura de este trabajo se pensó en que el especialista kinesiólogo es quien proporciona las directrices que el sistema debe tener en consideración al momento de monitorizar el tratamiento y el paciente al que se van a monitorizar sus movimientos, analizarlos y crear parámetros que generen un registro útil para el especialista.

Considerando que la gestión de la información del paciente es un conjunto de funciones que están a cargo del especialista kinesiólogo, y que los ejercicios excéntricos los realizará el paciente en su casa, se desarrollaron dos subsistemas: un módulo de gestión y monitoreo de pacientes, en donde el especialista kinesiólogo concentra las funciones de gestión y administración de la información de sus pacientes, y un módulo de adquisición de datos, módulo con el que el paciente interactúa con el sistema mediante el sensor de captura de profundidad KINECT. Ambos módulos se comunican entre sí por una base de datos, como se ilustra en la Figura 1.

\section{Subsistema de control y seguimiento de pacientes}

El módulo de gestión y monitoreo de pacientes, Figura 2, es un subsistema en el que una interfaz de interacción web permite al profesional de la salud realizar las tareas de gestión básicas sobre los datos de sus pacientes. Dichas tareas se pueden identificar como:

- Gestionar las fichas de los pacientes (datos personales y tratamientos).

- Realizar seguimiento del paciente.

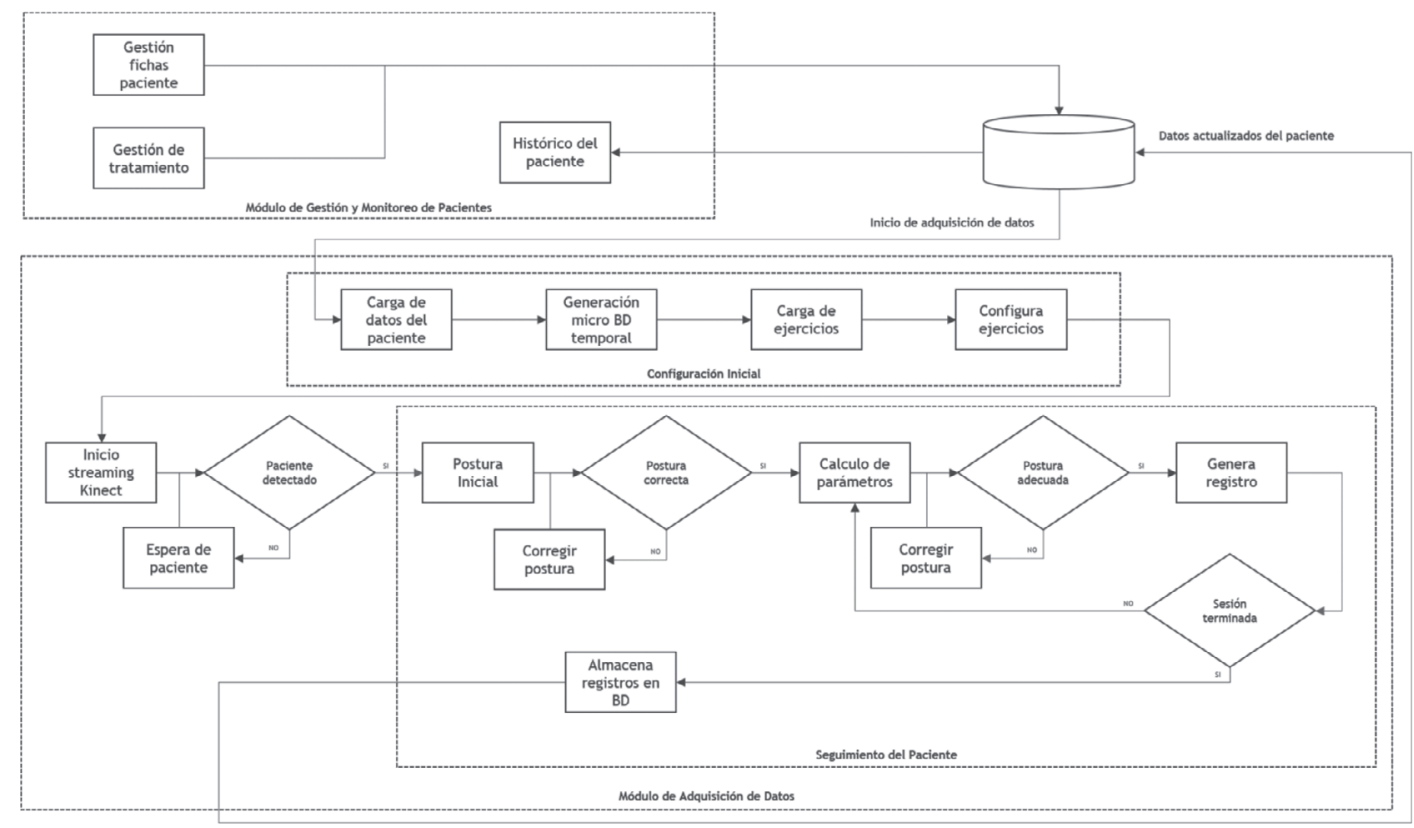

Figura 1. Arquitectura del sistema (fuente propia). 
Es importante señalar que al ser un módulo con interfaz web, se utilizó el modelo vista controlador para su diseño.

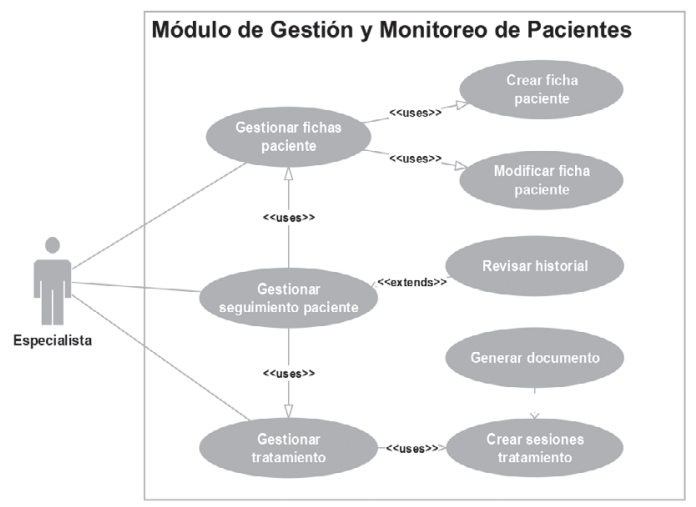

Figura 2. Diagrama de casos de uso del subsistema de control y seguimiento de pacientes (fuente propia).

\section{Subsistema de adquisición de datos}

Por otra parte, el módulo de adquisición de datos fue desarrollado para ser utilizado por el paciente. Este subsistema utiliza como hardware principal la cámara de profundidad KINECT.

La principal tarea de este módulo es capturar datos de los movimientos del paciente, estos serán analizados $\mathrm{y}$, dependiendo del comportamiento del paciente, se realizarán las correcciones correspondientes.

Este módulo se elaboró pensado en arquitectura por capas, como se ilustra en el diagrama de bloques de la Figura 3.

La primera capa se encarga de administrar la conexión con la base de datos. La capa de gestión de estados administra y provee los servicios del Framework XNA para todos los estados en los que se encuentre el módulo de adquisición de datos, como las pantallas de inicio, de pausa, de inicio de sesión, los menús, etc. La capa KINECT ofrece todos los servicios del sensor por medio de las API con las cuales se realiza el seguimiento del cuerpo del paciente y de esa forma obtener los puntos 3D de interés, de acuerdo con los ejercicios que él desarrolle. Con dichos puntos se crean los vectores que representan el cuerpo humano y se pueden obtener los ángulos que se forman con estos vectores. Finalmente cada uno de los resultados se procesa y se envía a la base de datos centralizada.

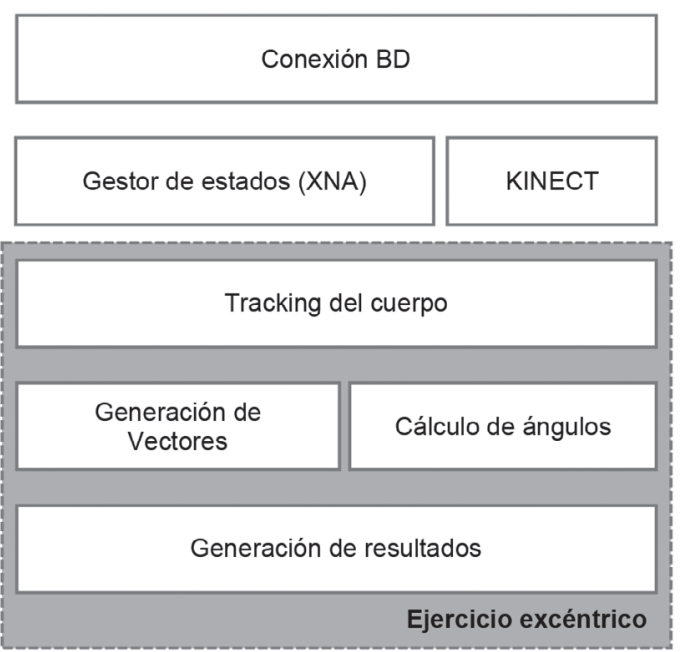

Figura 3. Diagrama de capas del subsistema de adquisición de datos (fuente propia).

Para generar los vectores y obtener los cálculos de los ángulos de cada gesto motor del paciente se utilizó el entorno de desarrollo (SDK) KINECT para Windows y XNA Framework y como lenguaje de programación $\mathrm{C \# .}$

\section{Dispositivo de captura de profundidad KINECT}

La principal innovación detrás de KINECT radica en que este dispositivo es capaz de realizar un mapeo de profundidad frente a cualquier objeto que interactúe con el sensor, dentro de un ambiente de iluminación controlada (ambiente con baja interferencia lumínica), sin requerir de una calibración (a excepción de la detección de la figura humana) [4].

KINECT [12], dentro de sus componentes principales, cuenta con una cámara RGB, un emisor infrarrojo y una cámara infrarroja, además de otros sensores.

En la Figura 4 se observa cómo es la cámara de profundidad KINECT y sus principales componentes: un proyector infrarrojo con el que el dispositivo proyecta un patrón luminoso, una cámara infrarroja donde se calcula el viaje del haz de luz infrarrojo emitido, y una cámara RGB con la que se captura una imagen en colores de lo que el dispositivo tiene en su rango de visión.

Para determinar la profundidad de los objetos frente al sensor KINECT realiza una telemetría [14] emitiendo un patrón de luz infrarroja y midiendo 


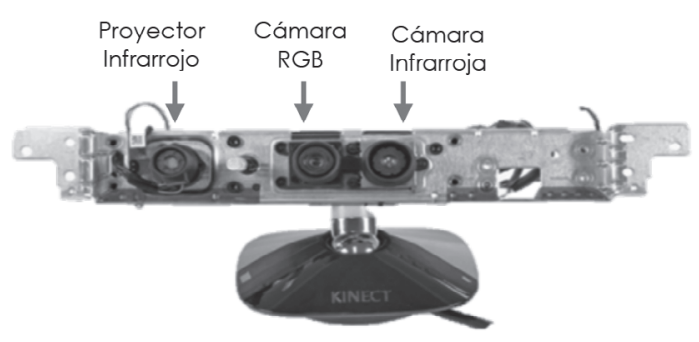

Figura 4. Componentes principales de KINECT [13].

el vuelo del haz de luz desde su emisión hasta su retorno.

En la Figura 5 se puede observar cómo el sensor KINECT emite un patrón luminoso, con el que realiza la telemetría y así determinar la distancia en profundidad de las personas u objetos que se coloquen frente al sensor.

Con los datos de profundidad se crea una imagen, a partir de la que se puede predecir dónde se encuentra la figura humana.

La Figura 6 ilustra una imagen de profundidad creada, a partir de los datos de profundidad que entrega el sensor.

Una vez que se crea la imagen de profundidad y se logra predecir la figura humana, es posible establecer un modelo anatómico basado en las principales articulaciones del cuerpo como coordenadas en 3D [4, 15], como se ilustra en la Figura 7.

No obstante, las coordenadas 3D de las articulaciones (joints) suelen tener cierto grado de imprecisión, por lo que, utilizando las imágenes de la cámara RGB y

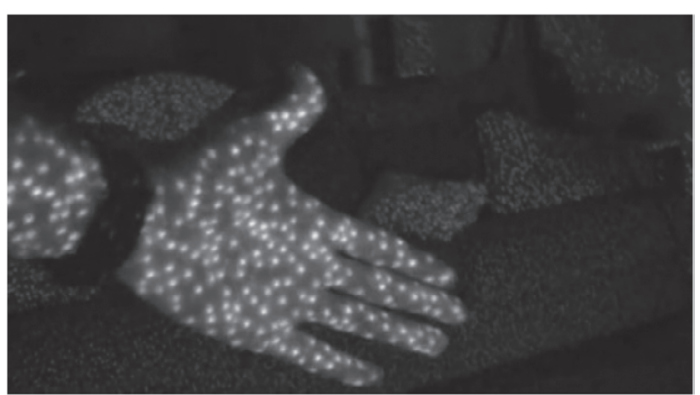

Figura 5. Patrón luminoso emitido por KINECT [13].

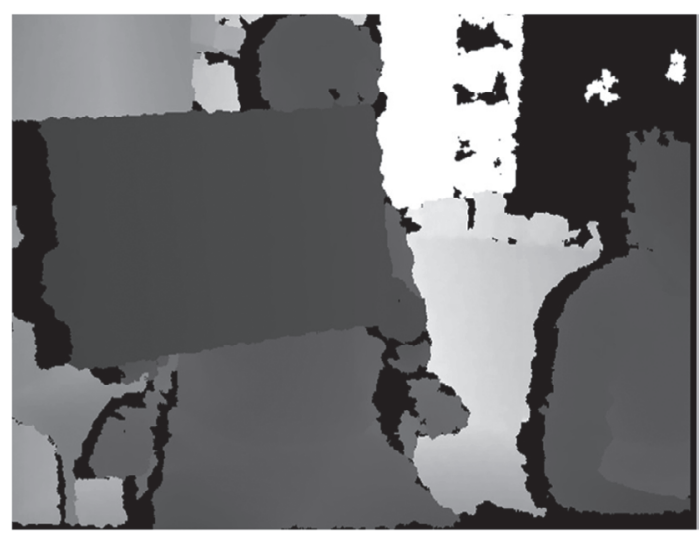

Figura 6. Imagen de profundidad creada con KINECT [4].

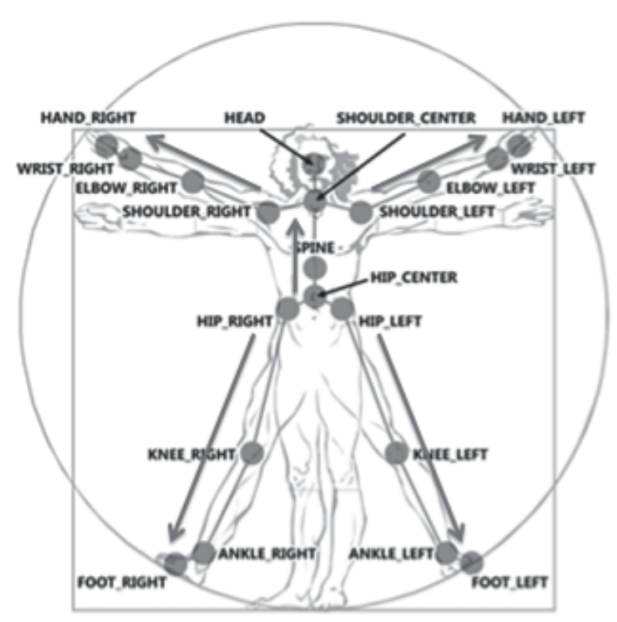

Figura 7. Modelo anatómico de acuerdo con KINECT [15].

la imagen creada a partir del mapeo de profundidad, se procede a realizar una calibración de cámara.

\section{Calibración de cámara}

KINECT puede modelarse siguiendo el modelo de cámara Pinhole descrito por Medioni y Kang [16] en la Figura 8.

De acuerdo con el modelo ilustrado en la Figura 8, $m=\left[\begin{array}{ll}u & v\end{array}\right]^{T}$ denota un punto 2D cualquiera. $m=\left[\begin{array}{lll}X & Y & Z\end{array}\right]^{T}$ denota un punto 3D.

En coordenadas homogéneas se representa como $\tilde{m}=\left[\begin{array}{lll}u & v & 1\end{array}\right]^{T}$ y $\tilde{M}=\left[\begin{array}{llll}X & Y & Z & 1\end{array}\right]^{T}$.

Según este modelo, la imagen de un punto 3D $M$ se forma por un rayo óptico desde $M$ pasando por 


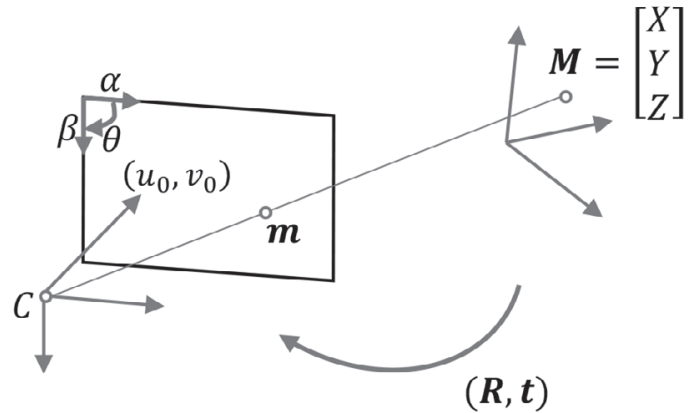

Figura 8. Modelo de cámara Pinhole [16].

el centro óptico $\mathrm{C}$ e intersecta el plano imagen en $\mathrm{m}$. Los tres puntos $C, M$ y $m$ son colineales. La relación entre el punto 3D $M$ y su proyección de imagen $m$ está dada por:

$$
\begin{gathered}
s \tilde{m}=\underbrace{A\left[\begin{array}{ll}
R & t
\end{array}\right]}_{P} \tilde{M} \equiv P \tilde{M} \\
\text { con } \quad A=\left[\begin{array}{lll}
\alpha & \gamma & u_{0} \\
0 & \beta & v_{0} \\
0 & 0 & 1
\end{array}\right] \\
y \quad P=A\left[\begin{array}{ll}
R & t
\end{array}\right]
\end{gathered}
$$

En donde $s$ es un factor de escala arbitrario $(R, t)$ llamado también parámetros extrínsecos, son la rotación y traslación que relaciona el sistema de coordenadas del mundo real con el sistema de coordenadas de la cámara, $A$ es la matriz intrínseca, con $\left(u_{0}, v_{0}\right)$ las coordenadas del centro de la imagen, $\alpha y \beta$ los factores que derivan el largo focal y tamaño de pixel en $u$ y $v, \gamma$ el parámetro que describe la asimetría de los ejes de la imagen. La matriz $P$ de $3 \times 4$ es la matriz de proyección de cámara, la que mezcla los parámetros intrínsecos y extrínsecos. En la Figura 8 el ángulo entre los ejes de imagen es denotado por $\theta$, por lo que se obtiene $\gamma=\alpha \cot \theta$. Si los pixeles son rectangulares, entonces $\theta=90^{\circ}$ y $\gamma=0$.

El objetivo de la calibración de cámara es determinar los parámetros de la transformación entre un objeto en el espacio 3D y la imagen 2D observada por la cámara.

\section{Calibración de Zhang}

En este trabajo se aprovecha la experiencia reportada en Aracena, Campos y Tozzi [17] donde se aprecia que el método de calibración de Zhang [18] entrega mejores resultados y se adapta para este tipo de aplicación.

Zhang propone un método de calibración basado en homografía. Este algoritmo requiere de por lo menos 4 puntos coplanares. De acuerdo con el modelo de cámara Pinhole, cuando todos los puntos 3D son coplanares, se selecciona el sistema de coordenadas globales de forma tal que el plano $Z$ es cero, con ello la ecuación (1) es posible reescribirla de la siguiente forma:

$$
\begin{gathered}
s \tilde{m}=A\left[\begin{array}{llll}
r_{1} & r_{2} & r_{3} & t
\end{array}\right]\left[\begin{array}{llll}
X & Y & 0 & 1
\end{array}\right]^{t} \\
s \tilde{m}=A\left[\begin{array}{lll}
r_{1} & r_{2} & t
\end{array}\right]\left[\begin{array}{lll}
X & Y & 1
\end{array}\right]^{t}
\end{gathered}
$$

Considerando $M^{\prime}=\left[\begin{array}{lll}X & Y & 1\end{array}\right]$ como un punto en el plano imagen, entonces es posible relacionar a $\tilde{m}$ y $M^{\prime}$ mediante una matriz de homografía $H$ :

$$
\begin{gathered}
s \tilde{m}=H M^{\prime} \\
\text { con } H=A\left[\begin{array}{lll}
r_{1} & r_{2} & t
\end{array}\right]
\end{gathered}
$$

Al definirse a lo menos 4 puntos no colineales en el modelo, la homografía $H$ puede ser determinada hasta un factor de escala que se puede escribir como:

$$
H=\left[\begin{array}{lll}
h_{1} & h_{2} & h_{3}
\end{array}\right]=\lambda A\left[\begin{array}{lll}
r_{1} & r_{2} & t
\end{array}\right]
$$

Siguiendo la ecuación (7) es posible descomponer la homografía $H$ para obtener la matriz intrínseca A, para ello se utiliza el método que Zhang [18] descrita en la sección 3.1 de su trabajo.

Gracias a ello se puede obtener la matriz de rotación $R$ y el vector de traslación $\mathrm{t}$ de la siguiente forma:

$$
\begin{aligned}
& r_{1}=\lambda A^{-1} h_{1} \quad \quad \begin{array}{r}
r_{2}=\lambda A^{-1} h_{2} \\
r_{3}=r_{1} \times r_{2}
\end{array} \quad t=\lambda A^{-1} h_{3} \\
& \text { donde } \quad \lambda=\frac{1}{\left|A^{-1} h_{1}\right|}=\frac{1}{\left|A^{-1} h_{2}\right|}
\end{aligned}
$$


Debido a la existencia de ruido en los datos, la matriz $R=\left[\begin{array}{lll}r_{1} & r_{2} & r_{3}\end{array}\right]$ no satisface, en general, las propiedades de una matriz de rotación, es por ello que en apéndice $\mathrm{C}$ de su trabajo Zhang describe un método para estimar la mejor matriz de rotación a partir de una matriz general de $3 \times 3$, de manera robusta.

\section{Tendones}

De los diferentes gestos motores que nuestro cuerpo es capaz de realizar, existen algunos más complejos de analizar que otros, debido a la cantidad de componentes que se ven involucrados al momento de la ejecución del gesto motor. Es por ello que para efectos de estudio este trabajo considera como foco de interés el tendón y sus lesiones.

\section{Lesiones de tendones}

Abellán [19] en su trabajo expone un resumen de los conceptos actuales en referencia a la clasificación de las lesiones tendinosas, las que se explican a continuación:

- Lesión por traumatismo directo o indirecto.

- Tendinopatía.

- Tendinosis.

- Tendinitis.

- Paratendinitis.

- Entesopatías.

\section{Tendón rotuliano}

El tendón rotuliano es el encargado de transmitir la fuerza de los cuádriceps hasta la tibia y permitir la extensión de rodilla. Es por ello que cuando este tendón se encuentra lesionado provocará dolor al momento de ejercer presión sobre el mismo, como por ejemplo en acciones como agacharse, patear, al subir y bajar escaleras, al saltar, etcétera.

La Figura 9 ilustra una vista anterior de la rodilla, en la que se puede apreciar los diferentes componentes de la misma, señalando específicamente dónde se encuentra el tendón rotuliano.

Las lesiones al tendón rotuliano, y otras como al tendón de Aquiles, son bastante frecuentes en el mundo deportivo [10], es por ello que gran parte de la actividad de los profesionales de las ciencias de la salud y la actividad física es en el tratamiento y prevención de estas lesiones, en especial aquellas provocadas por un mecanismo de sobrecarga.

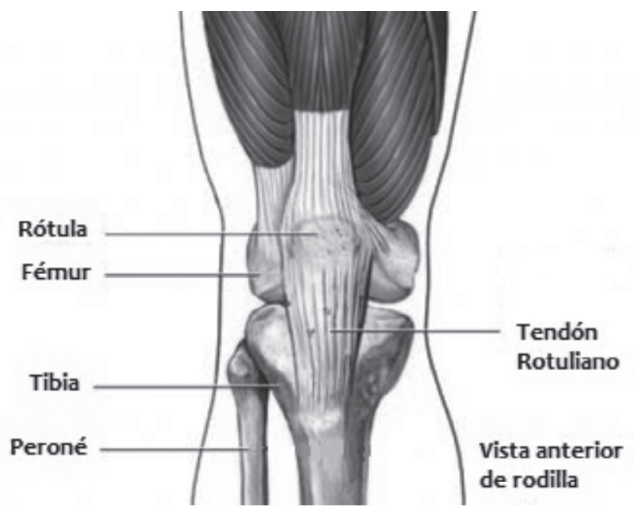

Figura 9. Tendón rotuliano [20].

Deportes como el vóleibol, baloncesto, atletismo o fútbol, tienden a afectar al tendón rotuliano, ya que son deportes que combinan gestos motores que requieren fuerza y velocidad en la extensión de la pierna.

La tendinosis del tendón rotuliano se caracteriza por un cuadro clínico con dolor en la cara anterior a la rodilla, que condiciona un déficit funcional.

\section{MATERIALES Y MÉTODOS}

Con la arquitectura de software propuesta se elaboró un prototipo de un sistema donde tanto el especialista kinesiólogo como el paciente poseen un módulo donde interactuar.

El kinesiólogo cuenta con el módulo de gestión y monitoreo de pacientes. Este módulo posee una interfaz de interacción web en donde el kinesiólogo tratante realiza la gestión tanto de las fichas de sus pacientes como de los tratamientos que ellos estén desarrollando.

En la Figura 10 se aprecia una vista de cómo el especialista puede llevar el control de sus pacientes. Es necesario destacar que para proteger la identidad de los sujetos de prueba la captura de pantalla fue realizada con datos ficticios a fin de ilustrar el sistema. Desde esta interfaz el kinesiólogo tratante puede establecer un protocolo que el paciente debe realizar en su casa. Dicho protocolo es generado en conjunto con el paciente tras varias consultas diagnósticas en la que el especialista debe instruir al paciente en cómo debe desarrollar los ejercicios de forma correcta. 


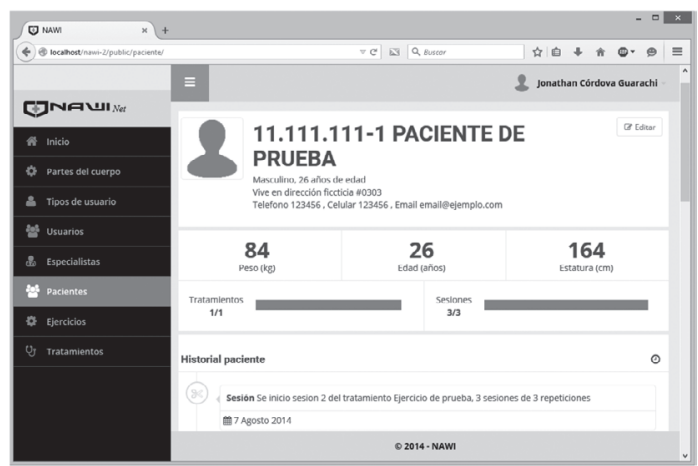

Figura 10. Interfaz web del módulo de gestión y monitoreo de pacientes (fuente propia).

Por su parte, el paciente tiene a su disposición el módulo de adquisición de datos, el que hace uso del sensor KINECT, con el que puede realizar los ejercicios que el kinesiólogo tratante haya definido e instruido como tratamiento en las consultas diagnósticas.

Una vez en su domicilio, el paciente utilizando sus credenciales deberá iniciar sesión en este módulo. $\mathrm{Al}$ hacerlo, el sistema automáticamente descarga los datos relacionados al paciente, reconstruyendo el protocolo de ejercicios de acuerdo con lo especificado por el kinesiólogo.

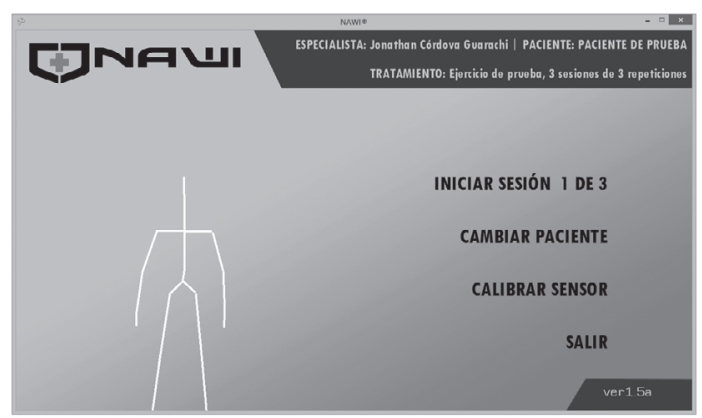

Figura 11. Interfaz del módulo de adquisición de datos (fuente propia).

Una vez seleccionado el inicio de su sesión, como se muestra en la Figura 11, el módulo buscará al paciente y verificará si se encuentra dentro de un área de trabajo, establecida entre 1,2 y 3,5 metros alejado del sensor (durante las pruebas se sugiere a 2 metros), como se ilustra en las siguientes figuras:

Las Figuras 12 y 13 dejan en evidencia cómo el sistema detecta al paciente dentro del área de trabajo

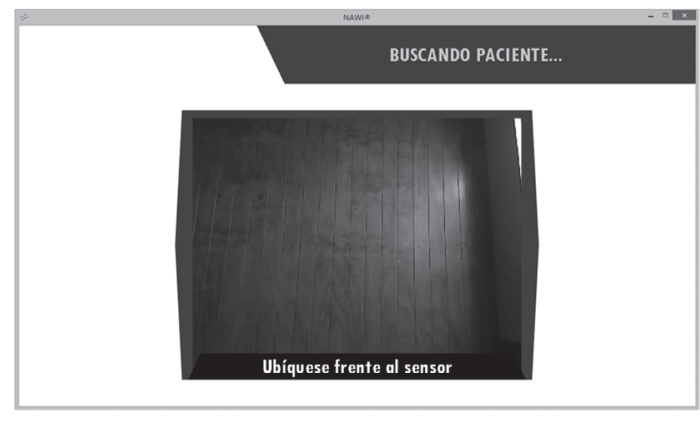

Figura 12. Sistema buscando al paciente (fuente propia).

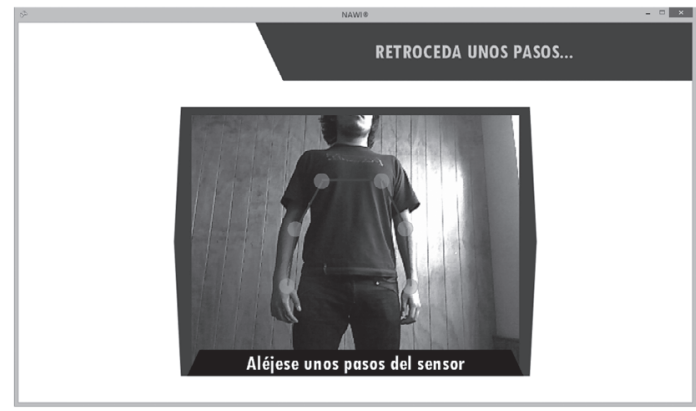

Figura 13. Sistema corrigiendo distancia del paciente (fuente propia).

y sugiere que cambie su ubicación de acuerdo con la distancia que existe entre el paciente y la cámara de profundidad, esto último se encuentra ilustrado en la Figura 14

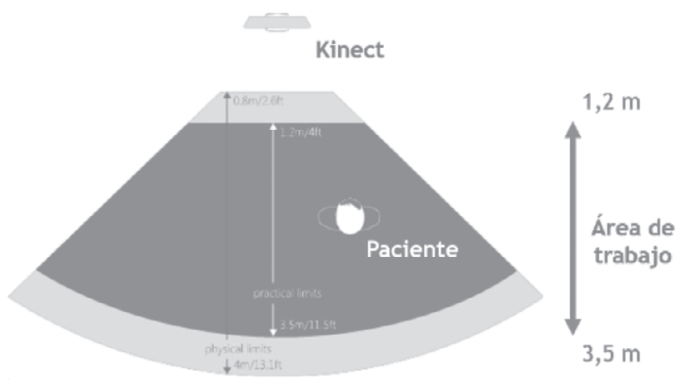

Figura 14. Área de trabajo de la cámara de profundidad [15].

Con el paciente en una posición adecuada, se procede a desarrollar los ejercicios excéntricos definidos.

Ejercicios excéntricos controlados desarrollados En el trabajo realizado se desarrollaron diferentes actividades basadas en ejercicios excéntricos, cuyo control varía desde la medición de los ángulos 
que el paciente crea, al desarrollar los ejercicios excéntricos, como del tiempo en que este tarda en ir desde una posición inicial a una posición final.

Para todos los ejercicios se solicita al paciente ubicarse de frente al sensor, para que se detecten todas las partes de su cuerpo. Luego, dependiendo de la actividad que el paciente deba realizar, el sistema entrega indicaciones para que pueda ubicarse de la mejor forma posible, dentro del espacio de trabajo.

Para el primer ejercicio se solicita al paciente que se ubique con su cuerpo girado, de manera tal que quede casi de perfil frente al sensor, de forma tal que la pierna afectada sea la que quede frente a KINECT. Luego con los brazos extendidos debe realizar una flexión de rodilla con la pierna que padece la tendinopatía intentando mantener, en lo posible, su espalda recta, como se ilustra en la Figura 15. El paciente puede apoyar sus manos en una superficie rígida para evitar perder el equilibrio durante el ejercicio.
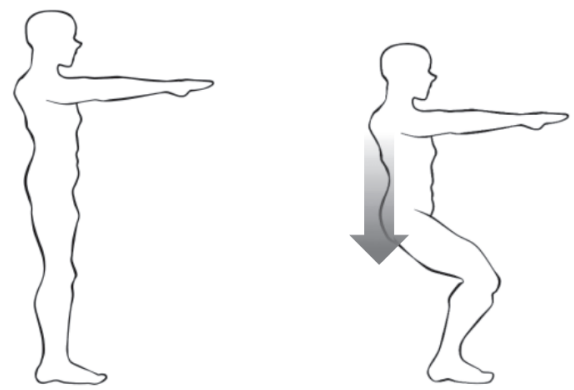

Figura 15. Flexión de rodilla (fuente propia).

Debido a que el ángulo de interés para este ejercicio radica en la rodilla, se considerarán los joints de la cadera, rodilla y tobillo para generar los vectores que permitirán obtener el ángulo formado en la rodilla, al momento de realizar la flexión.

Para generar los vectores se consideran como puntos vértices los joints calibrados mediante una correspondencia 2D-3D aplicando la ecuación (10)

$$
\tilde{m}=H M^{\prime}+\vec{t}
$$

Donde $\mathrm{m}, \tilde{m}, H$ y $M^{\prime}$ provienen de la ecuación (5) y $\vec{t}$ es un vector de corrección espacial, que permite superponer elementos virtuales sobre las imágenes generadas con KINECT, como se sugiere en la experiencia en Aracena [17] (ver Figura 16).

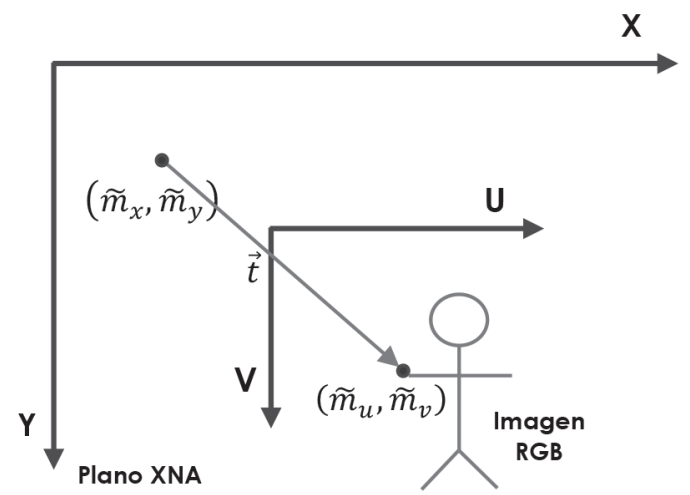

Figura 16. Correspondencia 2D-3D corregida (fuente propia).

Una vez generado dichos vectores se procede a calcular el producto escalar de los mismos y con ello aplicar la ecuación (11) y así obtener el ángulo deseado mediante trigonometría, donde $\vec{a}$ y $\vec{b}$ son los vectores generados y los que forman un ángulo de interés $\alpha$ entre sí.

$$
\cos \alpha=\left(\frac{\vec{a} \cdot \vec{b}}{|\vec{a}| \cdot|\vec{b}|}\right)
$$

El cálculo del ángulo permite modificar el nivel de la barra que se encuentra a la izquierda de la pantalla. Mientras mayor sea el ángulo calculado, mayor será la altura de la barra.

Indistintamente del ángulo alcanzado por el paciente, por cada repetición del ejercicio el sistema genera un registro, el que se le entrega al especialista para un análisis posterior.

Con este ejercicio se busca que el paciente realice flexiones cada vez más profundas, elevando la barra hacia un punto deseado, configurado previamente por el kinesiólogo tratante desde su propio módulo y señalado en la misma barra por una zona especial.

Es necesario destacar que cada ejercicio es regulado según la sensación de dolor que el paciente presente, por lo que si la sensación de dolor impide realizar la flexión, el sistema detectará qué ángulo alcanzó el paciente pasado un tiempo. De esta forma el sistema se regula solicitándole al paciente que alcance ángulos más cercanos a lo que físicamente puede ejecutar. 


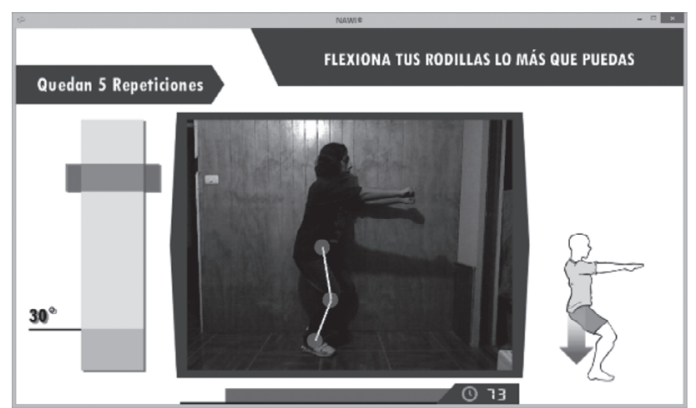

Figura 17.Ejecución de la flexión de rodilla (I) (fuente propia).

En las Figuras 17 y 18 se muestra cómo el paciente realiza la flexión de rodilla utilizando el sistema, el que le indica paso a paso cuales son los movimientos que debe realizar.

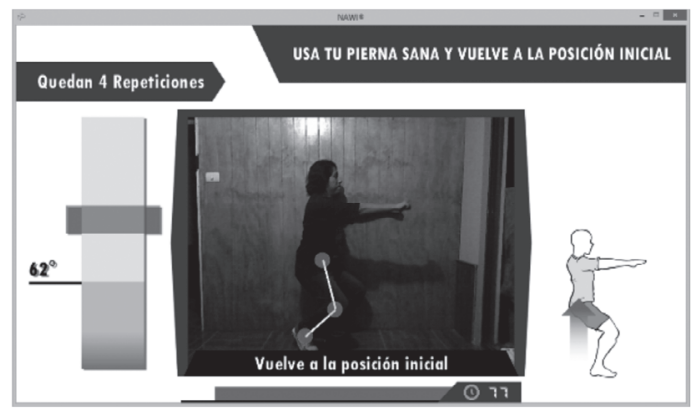

Figura 18. Ejecución de la flexión de rodilla (II) (fuente propia).

La flexión de rodilla posee diferentes variantes, como la que se presenta en las Figuras 19 y 20, en dicho ejercicio se busca aislar la presión sobre la rodilla afectada de la pierna que no presenta dolencia, dejando la pierna sana en el aire.

Posterior a la flexión, el paciente debe utilizar su pierna sana para volver a la postura inicial. Para ello el sistema emitirá una alerta sonora, para que el paciente inicie el movimiento de retorno al punto inicial. Esta alarma se emite tanto en el caso de que el paciente alcance el ángulo deseado como también en el caso de que el paciente no haya alcanzado lo solicitado.

En cada uno de los ejercicios, con ayuda de la correlación 2D-3D, es posible superponer unos discos y vectores sobre la pierna a controlar, lo que facilita al paciente corregir sus posturas en caso de que sea necesario. Para ello, en todo momento se
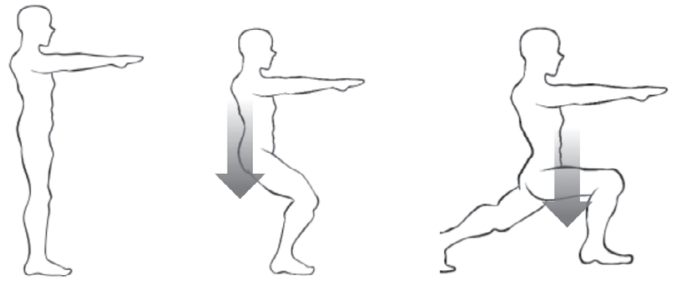

Figura 19. Flexión de rodilla con extensión de pierna sana (fuente propia).

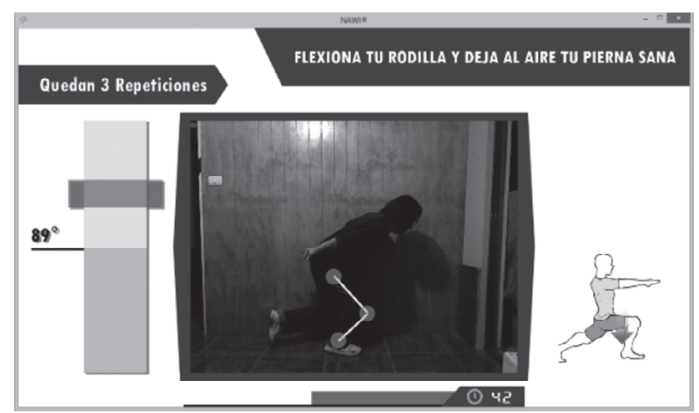

Figura 20. Ejecución de la flexión de rodilla con extensión de pierna sana (fuente propia).

determina la distancia entre las zonas del cuerpo del paciente y el sensor, estableciendo relaciones entre las posiciones de sus articulaciones durante la ejecución del ejercicio.

En la Figura 21 se muestra otro tipo de ejercicio excéntrico.
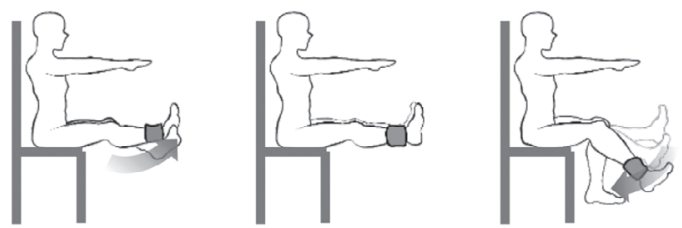

Figura 21. Flexión de rodilla usando contrapesos (fuente propia).

En este ejercicio se pide al paciente que flexione ambas piernas, utilizando la pierna que se encuentra sana como soporte para la pierna que padece de la tendinosis, para luego realizar una flexión de rodilla con la pierna sana, aislando la pierna afectada $y$, por ende, la fuerza que esta debe mantener. Posteriormente finalizar con una flexión de rodilla suave en la pierna que presenta la tendinosis, con la salvedad de que dicha pierna debe soportar un contrapeso que permita una mayor estimulación 
al tendón. La carga adosada al tobillo de la pierna puede ir variando según las indicaciones y control del especialista kinesiólogo tratante.

Para esta terapia se solicita al paciente que intente vencer el contrapeso por el mayor tiempo posible, es por ello que el sistema desarrollado mide el tiempo que el paciente vence la carga puesta en su tobillo mientras realiza el ejercicio.

Su ejecución se ilustra en la Figura 22.

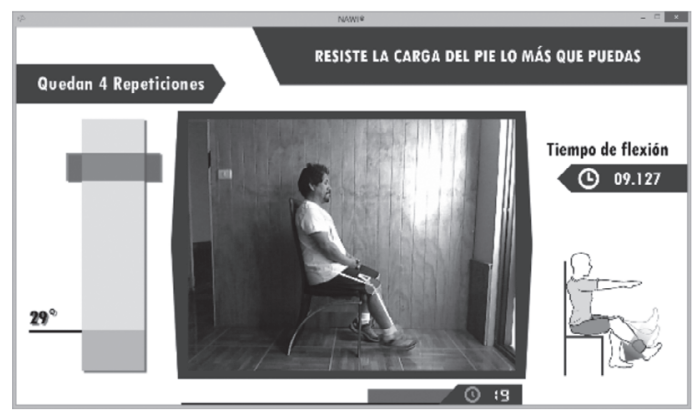

Figura 22. Ejecución de flexión de rodilla usando contrapesos (fuente propia).

Este último ejercicio puede ser desarrollado utilizando una máquina de musculación en el caso de disponer de ella, o utilizando pesas que se adapten al tobillo de la pierna afectada.

El objetivo de la aplicación es guiar al paciente a que alcance unas zonas azules a medida que realiza sus flexiones de rodilla. Estas zonas azules estarán limitadas por el mismo especialista al momento de crear el tratamiento del paciente desde el subsistema de control y seguimiento de pacientes. Estos parámetros son determinados posterior a un análisis inicial en conjunto con el paciente.

Cuando el paciente consigue alcanzar dichas barras, el sistema le solicitará que mantenga esa posición durante 5 segundos. Si lo consigue, se le indicará que la repetición fue desarrollada correctamente y se le solicitará que vuelva a su posición de inicio. De esta forma, el paciente puede descansar y prepararse para la siguiente repetición.

\section{Otros ejercicios excéntricos compatibles}

Es importante señalar que si bien se logró medir y controlar las repeticiones de flexiones de rodillas, existen variantes a este ejercicio excéntrico, las que pueden ser monitorizadas perfectamente por el módulo, como se ilustran algunos de ellos en las siguientes Figuras 23, 24 y 25.

La Figura 23 ilustra una flexión de rodillas en la que se busca solamente hacer presión sobre la rodilla afectada por la tendinosis, es por ello que dicho gesto motor se complementa con una abducción de la pierna que no presente la dolencia. Posteriormente, el paciente debe utilizar la pierna sana y ejercer fuerza con ella para levantar el cuerpo y volver a la postura inicial sin sobrecargar al tendón.
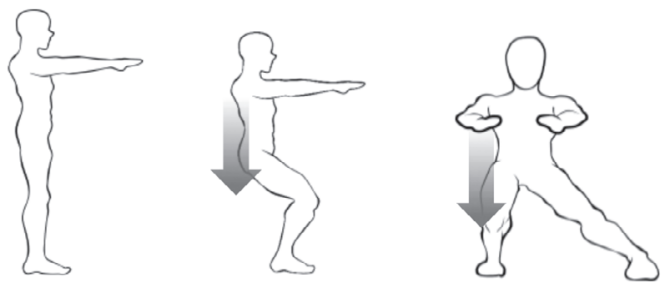

Figura 23. Ejecución de la flexión de rodilla con abducción de pierna sana (fuente propia).

En el ejercicio ilustrado en la Figura 24 se instruye al paciente que utilice un soporte en el talón de la pierna afectada, con el fin de generar un ángulo en el pie que puede variar entre los 25 a 35 grados. El paciente debe realizar una flexión de rodilla con la pierna afectada, mientras que la otra pierna queda suspendida en el aire, aislando el trabajo desarrollado solo a la pierna afectada. Luego el paciente regresa la pierna sana a la base y la utiliza para elevar el cuerpo y volver a su posición inicial.
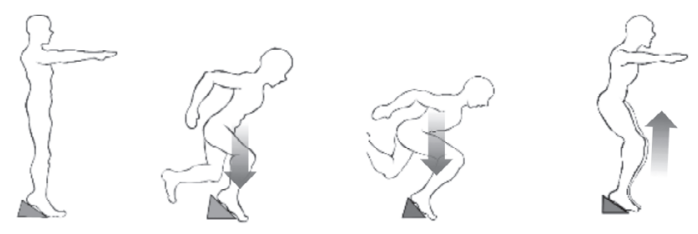

Figura 24. Ejecución de la flexión de rodilla con apoyo en solo un pie generando un ángulo de elevación (fuente propia).

La Figura 25 describe cómo un ejercicio puede apoyarse en el uso de una cinta elástica. Para ello el paciente deberá realizar una extensión con la pierna afectada de modo tal que mientras realiza la extensión debe vencer la carga impuesta por la cinta. Para ello deberá poner el pie de la pierna 
afectada en la cinta elástica, y suavemente realizar la extensión de la pierna.

Una vez que logra realizar la extensión, deberá dejar descansar la pierna afectada haciendo que la cinta lleve la pierna a su posición inicial.
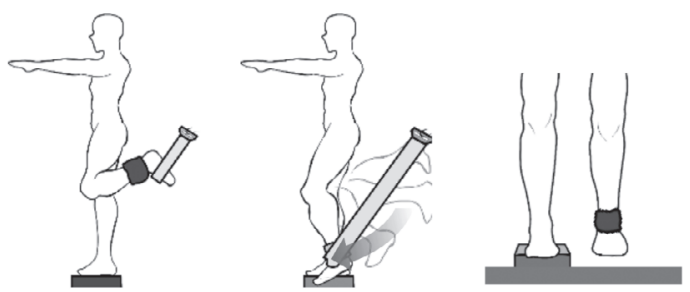

Figura 25. Extensión de rodilla contra resistencia (fuente propia).

A fin de validar los resultados que el sistema genera se realizaron pruebas clínicas en las que se comparó la precisión del sistema versus una técnica de medición tradicional utilizada por los kinesiólogos en ejercicio, en este tipo de terapias.

Para las pruebas se reunió un grupo de 30 voluntarios pertenecientes a un grupo etario de 24 a 30 años, a los cuales se les instruyó acerca del ejercicio con el que se sometieron a prueba con el sistema.

El ejercicio consistió en ejecutar un set de flexiones de rodilla frente al sensor. Antes de proceder a la ejecución del ejercicio a cada voluntario se le ajustó un goniómetro, un instrumento de medición tradicional. Mientras los voluntarios ejecutaban la flexión, el sistema registraba y calculaba el ángulo generado por la pierna. Al mismo tiempo, el goniómetro medía los grados del ángulo formado por la pierna del voluntario.

Por medio de un registro fotográfico ambas mediciones fueron generando una estadística (ver Figuras 26 y 27).

Esta experiencia se realizó 2 veces, la primera con el voluntario ubicado a 2 metros de distancia de KINECT y la segunda con el voluntario ubicado a 3 metros de distancia.

El objetivo de repetir la ejecución del ejercicio con estos dos criterios fue para determinar de forma empírica cuál es la distancia más idónea para realizar los ejercicios.

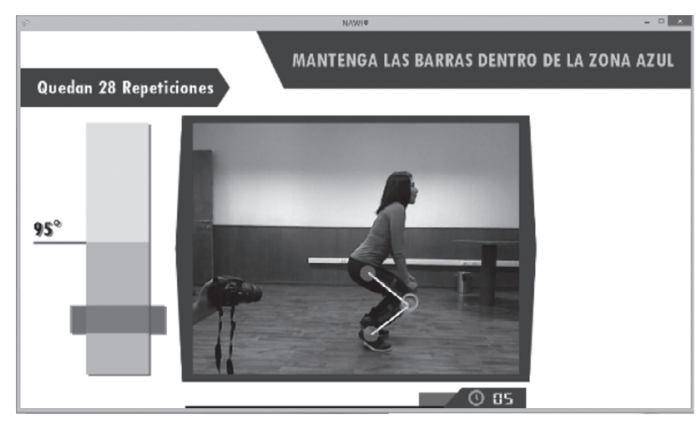

Figura 26. Participante realizando el ejercicio medido utilizando KINECT (fuente propia).

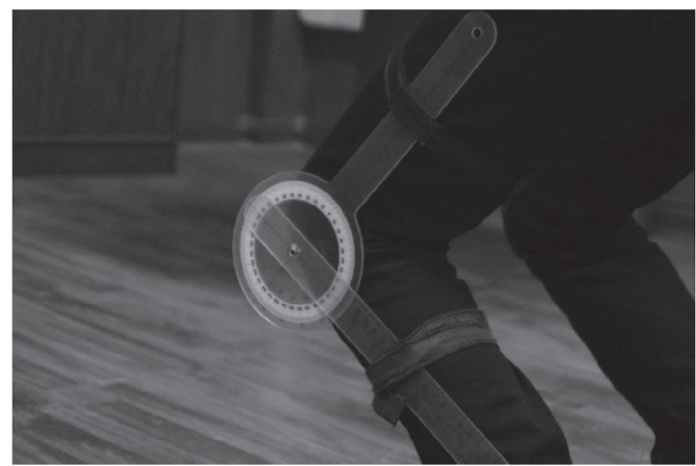

Figura 27. Participante realizando el ejercicio medido utilizando un goniómetro (fuente propia).

Los resultados de la primera experiencia en la que el voluntario se ubicó a 2 metros de distancia alejado del sensor se observan en la Figura 28.

En la Figura 28 se presentan las mediciones obtenidas con el sistema propuesto (columna negra) y las mediciones obtenidas con el instrumento tradicional de medición (columna gris) dentro de un gráfico cuyo eje vertical representa los grados alcanzados por las mediciones versus las muestras dispares significativas recolectadas durante la ejecución de las pruebas.

Se puede observar que en las diferentes flexiones realizadas por los voluntarios existe una ligera variación no superior a 10 grados entre ambas técnicas de extracción de datos. Es admisible destacar que en más de una oportunidad las mediciones con ambos instrumentos fueron exactas.

Del mismo modo al repetir la experiencia con el voluntario a 3 metros de distancia se construyó un segundo gráfico comparativo. 


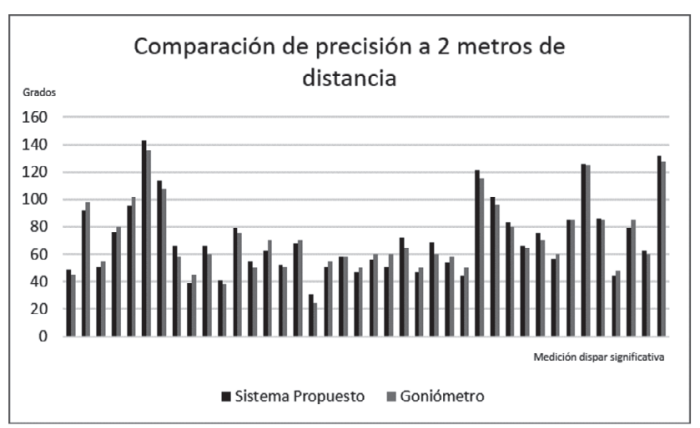

Figura 28. Gráfico comparativo entre KINECT a dos metros de distancia y un goniómetro (fuente propia).

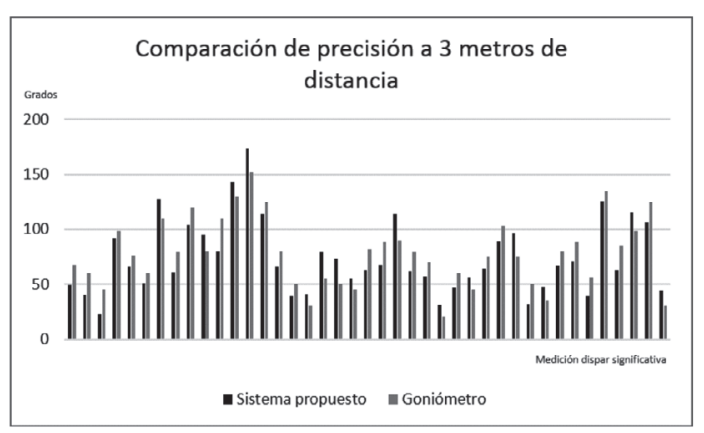

Figura 29. Gráfico comparativo entre KINECT a tres metros de distancia y un goniómetro (fuente propia).

La Figura 29 ilustra cómo la diferencia en la distancia a la que se ubicó al voluntario incidió en la precisión del sistema. Del mismo modo que en la prueba anterior, los datos se tabularon dentro de un gráfico cuyo eje vertical representa los grados alcanzados por las mediciones versus las muestras dispares significativas recolectadas durante la ejecución de las pruebas.

El registro realizado con el sistema (columna negra) presenta una variación mayor a los registros de la técnica tradicional de medición (columna gris). Dicha diferencia oscilaba entre los 20 a 30 grados.

\section{CONCLUSIONES}

Considerando los 2 escenarios de prueba, se deja en evidencia que el sistema desarrollado presenta una alta precisión, si el paciente se ubica a 2 metros de distancia en relación con KINECT, con lo que es posible inferir que el sistema entrega resultados aceptables con un mínimo margen de error. Esto a raíz de la variación poco significativa que presentó el sistema frente a un método tradicional de medición que los kinesiólogos realizan a sus pacientes.

El uso de la calibración de Zhang, corregida a la resolución del sistema desarrollado, permitió que el uso de elementos de realidad aumentada tenga una precisión aceptable.

Sistemas como el desarrollado no solo benefician al especialista kinesiólogo, el que cuenta con una herramienta de gestión de fichas y evolución del paciente, sino que además beneficia en su mayoría al paciente mismo, quien ya no requiere asistir de manera frecuente a la consulta del kinesiólogo, porque con el sistema desarrollado el paciente puede realizar, de forma controlada, los ejercicios en la comodidad de su hogar.

Esto se traduce en que el kinesiólogo puede monitorear de forma asíncrona el desarrollo del tratamiento del paciente, para posteriormente evaluar si el progreso amerita una modificación en la intensidad de los ejercicios, definir nuevos ejercicios o detectar de forma temprana alguna anormalidad durante el desarrollo del tratamiento, sin dejar de lado las consultas de control a las cuales el paciente debe recurrir.

Las pruebas realizadas con el sistema propuesto demuestran que es posible innovar en el quehacer del kinesiólogo, aportando con un sistema que presenta una precisión muy similar a la de los instrumentos utilizados en la práctica de su profesión. Más aún, durante las pruebas clínicas los voluntarios dieron sus puntos de vista en posibles mejoras para el sistema.

\section{TRABAJO FUTURO}

Se estima enriquecer la batería de ejercicios terapéuticos y de corrección de posturas, abriendo nuevas aristas a investigaciones paralelas que busquen diseñar aplicaciones lúdicas más efectivas no solo enfocada al tendón rotuliano, sino que abrir el espectro investigativo a otras partes del cuerpo humano, como la columna vertebral, las piernas, el codo, los hombros entre otras. 


\section{REFERENCIAS}

[1] Universia. "Kinesiología y Rehabilitación, Universidad de Tarapacá". Marzo 2014. URL: http://www.universia.cl/estudios/uta/ uta-kinesiologia-rehabilitacion/st/185914. Fecha de consulta: 20 de Octubre de 2014.

[2] E. Perandones Serrano. "Videojuegos en los hospitales: diseño e implementación de actividades y formación de educadores". Tesis para optar al grado de doctor. Universidad Complutense de Madrid. Madrid, España. pp. 132-322. 2011.

[3] Nintendo. "What is Wii". Febrero 2008. URL: http://web.archive.org/web/20080212080618/ http://wii.nintendo.com/controller.jsp. Fecha de consulta: 25 de marzo de 2014

[4] Z. Zhang. "Microsoft Kinect Sensor and Its Effect". MultiMedia, IEEE. Vol. $19 \mathrm{~N}^{\mathrm{o}} 2$, pp. 4-10. Abril 2012.

[5] E. Perandones Serrano. "Videojuegos para la Salud". VI Curso Comunicación y Salud nuevos escenarios y tendencias en tiempos de crisis. Madrid, España. Noviembre 2010.

[6] D. Freitas, A. Da Gama, L. Figuiredo, T. Chaves, B. Marques-Oliveira, V. Teichrieb and C. Araújo. "Development and Evaluation of a Kinect Based Motor Rehabilitation Game”. XI Simpósio Brasileiro de Jogos e Entretenimento Digital. Brasília, Brasil. Noviembre 2012.

[7] J.E. Muñoz Cardona, O.A. Henao Gallo y J.F. López Herrera. "Sistema de Rehabilitación basado en el Uso de Análisis Biomecánico y Videojuegos mediante el Sensor Kinect". TecnoLógicas, Edición Especia, pp. 43-54. Octubre 2013.

[8] C. Osorio Ochoa, J. Vázquez Valencia, J. Luna Córdova, A. Olvera López y M. Martín Ortiz. "Análisis de Marcha mediante Plataforma Kinect". La Investigación y las Aplicaciones en Ciencias de la Computación. FCC-BUAP. Puebla, México, pp. 17-21. 2011.

[9] J. Esteban Arango, J. Cárdenas Mazo y A. Peña Palacio. "Sistema para Rehabilitación del Síndrome del Miembro Fantasma utilizando Interfaz Cerebro-Computador y Realidad Aumentada”. Iberian Journal of Information Systems and Technologies. Vol. $6 \mathrm{~N}^{\circ}$ 11, pp. 93-106, 2013.
[10] F. Esperanza, F. Barrera, A. Pardo, J.F. Abellán, T. Fernández y L. M. Gonzáles. "Prevención de la tendinopatía rotuliana con ejercicios excéntricos en deportistas". Trauma. Vol. 22 No 4, pp. 241-247. 2011.

[11] D.C. Serpa Anaya. "Efecto del entrenamiento excéntrico sobre propiedades biomecánicas del tendón de aquiles". Tesis para optar al grado de doctor. Universidad de Granada. Granada, España. 2012.

[12] E. Naone. "Microsoft Kinect How the device can respond to your voice and gestures". Febrero 2011. URL: technologyreview. com/hack. Fecha de consulta: 17 de Abril de 2012.

[13] El Mundo. "El Gadgetoblog, Así se ve tu Kinect". Noviembre 2010. URL: http://www.elmundo.es/blogs/elmundo/ el-gadgetoblog/2010/11/08/asi-ve-kinecttu-salon.html. Fecha de consulta: 16 de Octubre de 2013.

[14] D. Beltrán Guerrero y L. Basañez Villaluenga. “Técnicas y algoritmos para la adquisición, transmisión y visualización de escenas 3D”. Universidad Politécnica de Catalunya. Barcelona, España. 2008.

[15] Microsoft. "Microsoft Developer Network". Mayo 2012. URL: http://msdn.microsoft. com/en-us/library/hh855347.aspx. Fecha de consulta: 20 de agosto de 2013.

[16] G. Medioni y S.B. Kang. "Emerging Topics in Computer Vision". Prentice Hall PTR. 2004.

[17] D. Aracena Pizarro, P. Campos y C.L. Tozzi. "Comparación de técnicas de calibración de cámaras digitales". Revista Facultad de Ingeniería. Universidad de Tarapacá. Vol. 13 $\mathrm{N}^{\circ}$ 1, pp. 57-67. 2005.

[18] Z. Zhang. "A Flexible New Technique for Camera Calibration". Marzo 1999. URL: http://research.microsoft.com/zzhang. Fecha de Consulta: 15 de septiembre de 2014.

[19] J.F. Abellán Guillén. "Terminología y clasificación de las tendinopatías". XX Jornadas Insternacionales de Traumatología del Deporte, Tendón y Deporte. Murcia, España. 2010.

[20] E. Tello Casadro. "Tendinitis Rotuliana". Marzo 2015. URL: http://www.fisioterapiaetc. com/tendinitis_rotuliana.html. Fecha de consulta: 30 de marzo de 2015. 